\title{
Educación Virtual Masiva Gratuita para la Prevención de Accidentes de Tránsito en Ambato
}

\author{
Education Free Virtual Mass for the Prevention of Road Accidents in Ambato
}

\author{
Fredy Maximiliano Jordán Cordone*, Raúl Armando Ramos Morocho ${ }^{\dagger}$, y Geovanny Eduardo Vega Villacis $\ddagger$ \\ Universidad Técnica de Babahoyo, Ecuador. \\ \{mjordan,rramos,gvega\}@utb.edu.ec
}

Fecha de recepción: 4 de agosto de 2016 — Fecha de aceptación: 16 de agosto de 2016

\begin{abstract}
Resumen-Los accidentes de tránsito es una problemática de tipo mundial, la Organización Mundial para la Salud en su informe 2009 señala que son más 1,2 millones de muertos al año por esta causa, también indica que la prevención de los accidentes de tránsito implica una amplia gama de medidas donde la educación es un pilar fundamental. En Ecuador la situación es igual, para el 2014 se reportaron más de $\mathbf{3 5 0 0 0}$ incidentes de tránsito El presente proyecto propone un proceso de capacitación virtual masiva en aspectos relacionados con leyes de tránsito, señales y estadísticas para lograr así una concientización entre los conductores, y en base a ello reducir los índices de accidentes. Se ha tomado como caso de estudio, las escuelas de conducción de los Sindicatos de Choferes profesionales de Tungurahua, se tuvo una población a investigar conformada por alumnos de estos centros de capacitación, dicha población asciende a 1600 personas, se trabajó con una muestra que llego a 350 personas. Del estudio realizado en base a encuestas, se concluyó que existen diversos factores para los accidentes de tránsito, pero que los más frecuentes son: el exceso de velocidad, el conducir en estado etílico, la poca precaución y el cansancio, también se concluyó que todas las personas están dispuestas a recibir procesos de capacitación de forma presencial o virtual. Luego del estudio, se implementó la MOOC ubicada en la dirección http://cursos.cedia.org.ec/, se matriculó alrededor de 500 personas y se las capacitó. El proyecto se complementó con diversos accesos a la plataforma como aplicaciones móviles, afiches promocionales con códigos QR y más.
\end{abstract}

Palabras Clave-Accidentes, Transito, Educación, Virtual, Capacitación.

Abstract-Traffic accidents is a problem of a global nature, the World Health Organization in its 2009 report notes that are 1.2 million deaths a year from this cause, also indicates that the prevention of traffic accidents involves a wide range of measures where education is a fundamental pillar. In Ecuador the situation is the same, by 2014 more than 35000 traffic incidents were reported This project proposes a process of massive virtual training on issues related to traffic laws, signs and statistics in order to achieve an awareness among drivers, and based on this reduce accident rates. Is taken as a case study, driving schools Trade Union of Professional Drivers of Tungurahua, a population had to investigate comprising students of these training centers, this population is 1600 people, we worked with a sample that arrived 350 people. The study based on surveys, it was concluded that there are several factors to traffic accidents, but the most common are: speeding, driving inebriated, little caution and fatigue, it was also concluded that all people are willing to receive training processes in person or virtual. After the study, the MOOC was implemented located in the http://cursos.cedia.org.ec/ direction, around 500 people enrolled and trained them. The project was supplemented by various accesses to the platform and mobile applications, promotional posters with QR codes and more.

Keywords-Accidents, Traffic, Education, Virtual, Training.

\section{INTRODUCCIÓN}

$\mathbf{L}$ a principal característica de los finales del siglo XX y principios del XXI es el gran desarrollo tecnológico en áreas como la electrónica, la informática y las telecomunicaciones. Todas estas tecnologías cada día están influenciando más de manera directa en la educación y han generado nuevos paradigmas educativos que solventan problemas como el de la distancia y tiempo. La educación virtual o E-Learning es definida por Rosenberg (2001) como: el uso de tecnologías y del Internet para la entrega de un amplio rango de soluciones que mejoran el conocimiento y el rendimiento."

OMS (2009) señala en su informe que: "Todos los años fallecen más de 1,2 millones de personas en las vías de tránsito del mundo, y entre 20 y 50 millones sufren traumatismos

\footnotetext{
*Licenciado en Sistemas Computacionales, Magister en Informática Empresarial.

${ }^{\dagger}$ Ingeniero en Sistemas, Magister en Informática Aplicada.

$\ddagger$ Ingeniero en Sistemas, Magister en Conectividad y Redes de Ordenadores.
}

no mortales. En la mayoría de las regiones del mundo, esta epidemia de accidentes de tránsito sigue aumentando. Los países de ingresos bajos y medianos tienen tasas más altas de letalidad por accidentes de tránsito (21,5 y 19,5 por 100000 habitantes, respectivamente) que los países de ingresos altos (10,3 por 100000$)$. Más del $90 \%$ de las víctimas mortales de los accidentes de tránsito que ocurren en el mundo corresponde a países de ingresos bajos y medianos, que tan sólo tienen el $48 \%$ de los vehículos del mundo. Cerca de la mitad de las personas que fallecen como consecuencia de accidentes de tránsito son peatones, ciclistas o «usuarios de vehículos de motor de dos ruedas"

OPS (2011) en su informe sobre "Traumatismos causados por el tránsito" consideran que la prevención de los traumatismos causados por el tránsito comprende una gama amplia de medidas donde el pilar fundamental es la educación.

En el Ecuador, ANT (2014) manifiesta que en el año 2013 se tuvieron 19330 accidentes de tránsito de los cuales el $3.06 \%$ se dieron en la provincia del Azuay. Un informe a Noviembre 
del 2013 señala que existieron 2050 fallecidos por accidentes de tránsito a nivel nacional correspondiéndole a la provincia del Azuay un 2, 53\% mientras que los lesionados por estos accidentes alcanza un número de 22651 correspondiéndole a la provincia un $3,35 \%$. La misma entidad estatal señala que para el primer semestre del 2014 el número de fallecidos llego a 1276 personas mientras que el número de accidentadas está en 14612, se puede apreciar un incremento importante de un año a otro. De estas estadísticas se obtiene que en el $60 \%$ de los accidentes de tránsito fatales, están involucrados choferes profesionales.

Por otro lado, a pesar del endurecimiento en leyes y sanciones los niveles de accidentes se elevan especialmente por los factores velocidad y ebriedad, esto quiere decir que son factores resultantes de la poca concientización respecto a la responsabilidad que tienen al conducir. Este problemática se deriva de un bajo nivel cultural en cuanto a prevención. Complementariamente a esto, muchos conductores desconocen ciertas señales de tránsito, así como las últimas penalidades asignadas en los nuevos estamentos legales.

Considerando estas situaciones conflictivas, los investigadores proponen como aporte de solución a la problemática, la ejecución de un proyecto donde se pueda capacitar de manera masiva y de forma on-line a un elevado número de personas que estén formándose para ser choferes profesionales. Para ello se contacta con las escuelas de conducción de los Sindicatos de Choferes profesionales de Tungurahua y surge el proyecto denominado "Capacitación masiva online" como un factor de solución a esta problemática.

El proyecto plantea el siguiente objetivo general: Emitir una capacitación virtual masiva gratuita orientada a los estudiantes de los Sindicatos de Choferes profesionales de la provincia de Tungurahua, la cual utilice la Red CEDIA como medio de enlace y contenga aspectos relacionados a prevención de accidentes de tránsito, leyes y más, para que en base a ella, se logre mayor concientización en los futuros choferes profesionales con respecto a la precaución que se debe tener durante la conducción de un medio de transporte y así evitar accidentes.

\section{Como fundamento teórico del presente trabajo investigativo tenemos:}

La Educación Virtual canaliza el uso de las nuevas tecnologías, hacia el desarrollo de metodologías alternativas para el aprendizaje de alumnos de poblaciones especiales que están limitadas por su ubicación geográfica, la calidad de docencia y el tiempo disponible (Bartolomé, 2004).

Los entornos virtuales se han ido popularizando en el presente siglo, aparecieron a finales del anterior y una de las plataformas más representativas es Moodle. Existen otras que también han repuntado en su uso, así tenemos por ejemplo Blackboard y Dokeos (William and Rice, 2009).

La plataforma Moodle es una aplicación web encuadrada dentro de lo que se conoce hoy como "Sistemas de Gestión de Aprendizaje" (LMS, Learning Management Systems) también conocidas como plataformas e-learning, un subgrupo de los gestores de contenidos. Otro termino también utilizado para para esto es "Entorno virtual de aprendizaje" (del Rosario Sánchez et al., 2009).

La formación en este siglo XXI se está orientando hacia nuevos modelos de enseñanza masivos en abierto y gratuitos. Estos modelos interactivos, colaborativos y online aumentan y posibilitan el acceso a la formación superior de manera universal. El movimiento conocido con la sigla inglesa MOOC (massive open online course) se basa en plataformas de aprendizaje dirigido desde los principios de ubicuidad, autoevaluación, modularidad y/o simulación.

El término MOOC fue acuñado en 2008 por Dave Comier de la University of Prince Edward Island en respuesta a un curso llamado Conectivismo y conocimiento Conectivo dictado por George Siemens de la Athabasca University y Stephen Downes del National Research Council (Universidad Carlos III, 2009). Consistió de 25 estudiantes con matrícula paga en Educación Continuada de la University of Manitoba, así como alrededor de 2200 estudiantes en línea provenientes del público general que no pagaron nada. Todo el contenido del curso estuvo disponible en la web y los estudiantes en línea pudieron participar a través de herramientas colaborativas. En 2011 más de 160.000 personas se matricularon en un curso de Inteligencia artificial ofrecido por la Universidad de Stanford.

Según La Hora (2013) en su artículo "Prevención de accidentes de tránsito se inició en Colegios" se manifiesta que: "Tras el lanzamiento de la campaña "No a los accidentes de tránsito, más respeto a la vida", impulsada por el Consejo de la Judicatura, Policía Nacional y la Fiscalía Provincial, ayer inició la primera capacitación dirigida a cerca de 200 estudiantes del Instituto San Francisco de la ciudad de Zamora. "No más accidentes de tránsito en Zamora Chinchipe" es el mensaje que los capacitadores le ponen énfasis para llegar a los estudiantes, debido al alto índice de accidentabilidad registrado en la provincia, manifestó el sargento segundo Wilman Sisalima, capacitador.

\section{DESARROLLO}

\section{Materiales y Métodos}

Los investigadores adoptaron el paradigma cuantitativo como modalidad de investigación. La investigación a desarrollar es aplicada y dentro de los tipos de investigación utilizadas son:

- De campo: llevada a cabo en base a encuestas a los estudiantes de las escuelas de conducción:

- Documental: Utilizada para elaborar el marco teórico. Para iniciar la investigación de campo primeramente se define el universo a investigar, en este caso dicho universo estuvo compuesto por los estudiantes de las escuelas de conducción de Tungurahua.

Tabla 1. Población a investigar

\begin{tabular}{|c|c|}
\hline Sindicatos & Alumnos \\
\hline ,Ambato & 1200 \\
\hline Baños & 100 \\
\hline Cevallos & 100 \\
\hline Mocha,Patate, Pelileo & 150 \\
\hline Píllaro,,Quero, Tisaleo & 150 \\
\hline Total & 1700 \\
\hline
\end{tabular}

Fuente: Escuelas de conducción de Tungurahua. 
La muestra se obtuvo en base a la siguiente fórmula:

$$
\text { Muestra }=\frac{\text { Poblacion }}{(\text { Poblacion }-1) * \text { error }^{2}+1}
$$

Se asumió un error del 4,6\%

$$
\begin{aligned}
\text { Muestra } & =\frac{1700}{(1700-1) * 0,046^{2}+1} \\
\text { Muestra } & =366
\end{aligned}
$$

La técnica aplicada fue: Encuesta a estudiantes. El instrumento utilizado fue el cuestionario. A continuación el diseño del cuestionario.

Cuestionario para los estudiantes de los Sindicatos de Choferes de Tungurahua.

1. ¿Crees tú que en el Ecuador, los índices de los accidentes de tránsito en los últimos años han disminuido? Totalmente (...) Parcialmente (....) Todo lo contrario, han aumentado (...)

2. ¿A qué factores crees que se deba que en la gran mayoría de accidentes de tránsito graves, estén inmersos choferes profesionales? Poca precaución (... Exceso velocidad (....) Cansancio por exceso de trabajo (...)

3. ¿Cuál de estas causas te parece que inciden más en los accidentes de tránsito?

Poca precaución (...) Exceso velocidad (....) Cansancio por exceso de trabajo (...)

4. ¿Qué nivel de incidencia tiene el exceso de velocidad en los accidentes de tránsito? Gran Incid. (...) Mediana Incid. (...) Poca Incid. (...) Ninguna Incid. (...)

5. ¿Cuál es tu nivel de conocimiento respecto a las sanciones por infracciones de tránsito? Elevado (....) Medio (....) Bajo (......) Muy Bajo (.....)

6. ¿Cuál es tu nivel de conocimiento respecto a las señales de tránsito? Elevado (....) Medio (.....) Bajo (.....) Muy Bajo (.....)

7. ¿Qué factor crees tú que pude influenciar en la reducción de los accidentes de tránsito?

Concientización ciudadana $(. . . \ldots)$ Elevación de sanciones $(. . . . .$.

8. ¿Cree usted que la concientización ciudadana se puede lograr en base a capacitación?

Totalmente de acuerdo (... ) Parcialmente de acuerdo (....) En total desacuerdo (...)

9. ¿Estaría usted dispuesto a capacitarse en estos aspectos para colaborar en la reducción de los accidentes de tránsito?

Totalmente de acuerdo (... ) Parcialmente de acuerdo (....) En total desacuerdo (...)

10. ¿De qué forma te gustaría capacitarte?

Presencial (....) Virtual (.....)

El instrumento fue validado por Directivos de las escuelas y por Docentes de varias universidades como Uniandes y la Universidad Técnica de Ambato

\section{RESULTADOS}

\begin{tabular}{|c|c|c|c|c|c|}
\hline \multirow{2}{*}{ Preguntas } & \multicolumn{4}{|c|}{ Respuestas } & \\
\hline & Resp.1 & Resp.2 & Resp.3 & Resp.4 & Total \\
\hline Una & 38 & 256 & 72 & & 366 \\
\hline Dos & 76 & 216 & 74 & & 366 \\
\hline Tres & 94 & 220 & 52 & & 366 \\
\hline Cuatro & 274 & 78 & 14 & & 366 \\
\hline Cinco & 76 & 242 & 40 & 8 & 366 \\
\hline Seis & 90 & 210 & 66 & & 366 \\
\hline Siete & 296 & 74 & & & 370 \\
\hline Ocho & 294 & 70 & 2 & & 366 \\
\hline Nueve & 296 & 66 & 4 & & 366 \\
\hline Diez & 208 & 158 & & & 366 \\
\hline
\end{tabular}

Los resultados de la investigación a los estudiantes son:

Tabla 2. Tabulación de la encuesta a los estudiantes

Fuente: Elaboración propia.

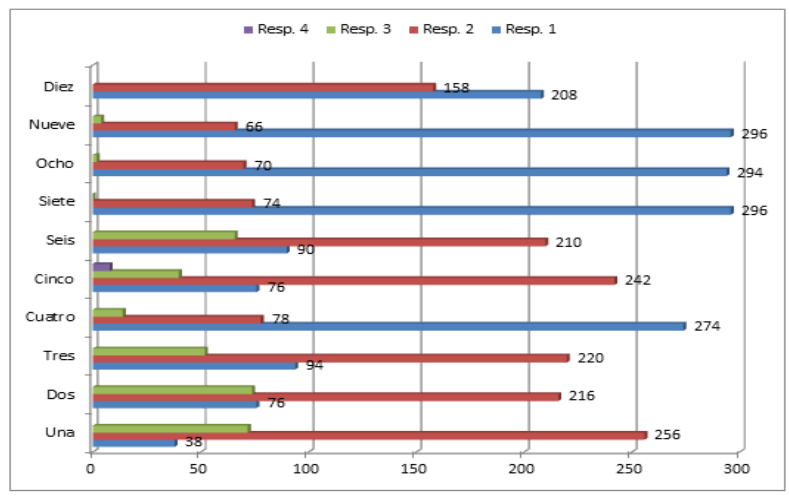

Figura 1. Tabulación de resultados encuesta alumnos.

Fuente: Elaboración propia.

\section{Conclusiones de la investigación de campo:}

- Se tienen medianos conocimientos sobre leyes de tránsito y reconocimiento de señales.

- Los niveles de concientización son relativamente bajos.

- La gran mayoría concuerda en que los procesos de capacitación ayudaran a elevar los niveles de concientización de los choferes con relación a la prevención de los accidentes de tránsito.

- Se ha determinado que existe una gran preferencia por la capacitación virtual y mixta relacionada con estos aspectos preventivos de los accidentes de tránsito

- La gran mayoría está dispuesta a capacitarse para prevenir accidentes de tránsito.

\section{Desarrollo del curso virtual masivo.}

Para el desarrollo del curso virtual masivo se llevaron a cabo las siguientes actividades previas:

\section{Definición de contenidos:}

Los contenidos fundamentales del curso virtual masivo fueron:

- Información general sobre accidentes de transito

- Informes de la OPS y el Banco Mundial 
- Los accidentes d etránsito a nivel mundial

- Los accidentes de tránsito a nivel nacional

- Leyes de transito

- Resumen general sobre las principales leyes de tránsito

- Sanciones a infractores

- Severidad d elas leyes

- Señales de tránsito

- Principales señales de transito

- Concientizando sobre accidentes de tránsito

- Lo que NO queremos que pase

\section{Implementación del curso virtual masivo}

Se procedió a la construcción del curso con los contenidos seleccionados, la direccion del mismo es :

https://cursos.cedia.org.ec/course/view.php?id=19.

Se ha considerado que el aula virtual debe ser lo mas agradable posible de tal manera que el estudiante se sienta a gusto durante el proceso de capacitación virtual. Es por ello que se ha incorporado el tema de moodle adquirido por CEDIA. También se ha incoporporado el plugin sidebar para lograr la elaboración de una aula iconografica que es un diseño totalmente novedose en moodle.

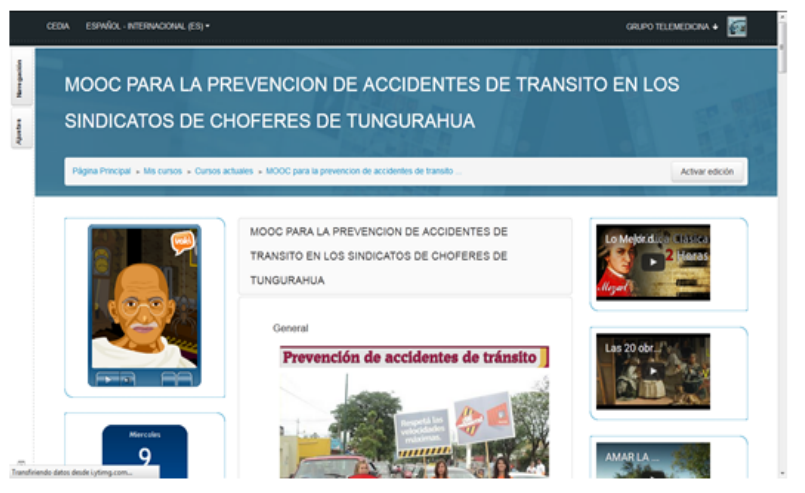

Figura 2. Pantalla inicial del curso virtual masivo.

Fuente: Elaboración propia.

\section{Evaluación}

Todos los test de evaluación previa y posterior al curso son automáticos y fueron generados con el software hotpotatoes.

\section{Configuración de Acceso.}

Se ha considerado importante que la MOOC pueda ser accesada de diversas maneras, es decir no solamente desde un computador sino más bien desde dispositivos móviles como teléfonos y tabletas. Para ello primeramente se debió configurar al sitio para que se adapte automáticamente al tamaño del dispositivo en el cual está siendo cargado. Esto se ha logrado mediante la configuración de un tema de carácter responsivo. (Figura 3).

\section{Generación del codigo $Q R$.}

Este elemento permite una carga rápido del sitio a traves de los dispositivos móviles. Ilustración No 3. Carga del curso en un dispositivo móvil y código Qr del curso.

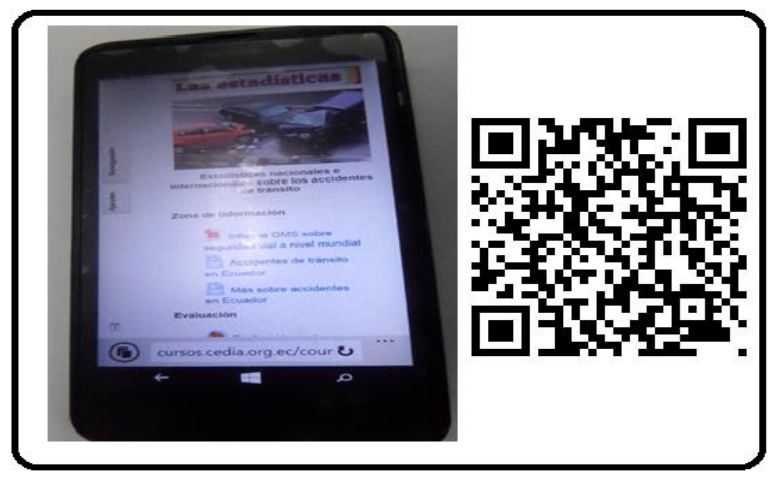

Figura 3. Carga del curso en un dispositivo móvil y código Qr del curso.

Fuente: Elaboración propia.

\section{Ejecución de la MOOC con las Instituciones provinciales.}

Luego de la contrucción del curso vitrual en la plataforma de CEDIA este debía ser ejecutado con los estudiantes de los sindicatos, previamente a ello se hicieron algunas actividades como:

\section{Diseño de la promoción del curso.}

Se trabajó para generar afiches promocionales de la Mooc y para que su acceso sea mas fácil y rapido

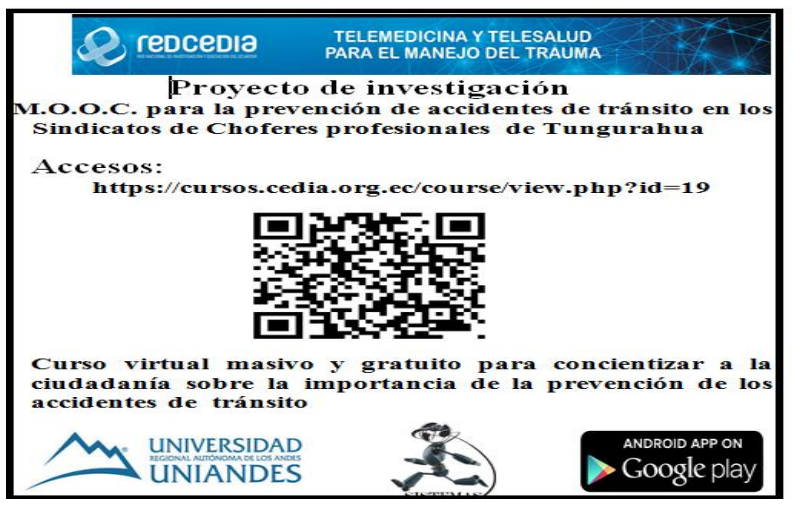

Figura 4. Poster promocional del curso con el enlace y código Qr Fuente: Elaboración propia.

Se considero importante facilitar enormenente el acceso al curso y para ello se trabajo en el desarrollo de una aplicación móvil que permita dicho acceso rápido en dispositivos con tecnología Andrdoid (Buscar por cyssacedia en Google Play). 


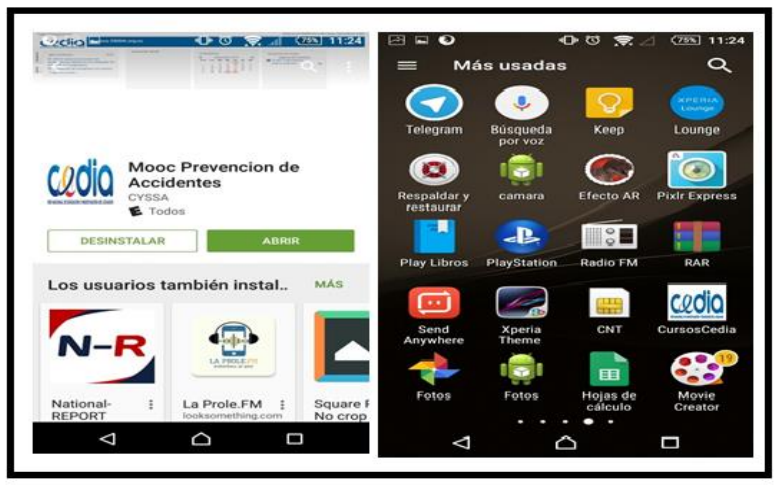

Figura 5. Capturas de la aplicación móvil desarrollada.

Fuente: Elaboración propia.

Se consideró importante un acceso automático al curso, para ello se requirió un registro facil, este se logró mediante un formulario en Google Docs.

Link:

https://docs.google.com/forms/d/1FZozcPilLOIPncos9pDaqZLoq HXzxA7dJRaZVY6mnNM/viewform

Se creyo conveniente la creación de un sitio Facebook para promocionar la Mooc y captar usuarios externos en base a un link que cargue el formulario de inscripción.

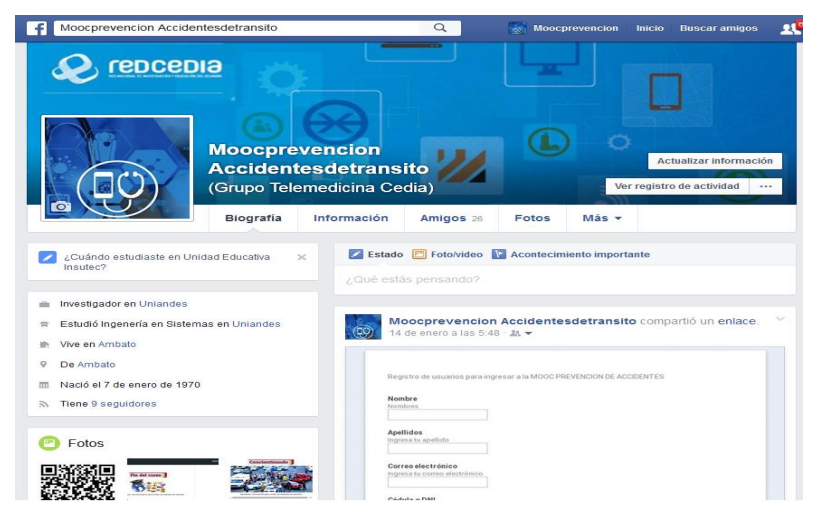

Figura 6. Sitio en Facebook del curso virtual Fuente:Elaboración propia.

Luego de todas las actividades promocionales se procedió a la ejecución de la MOOC en las escuelas de conducción. Se hicieron reuniones demostrativas del acceso al curso y se lo llevo a cabo en las principales escuelas de conducción. Debe señalarse que se hizo una evaluación inicial antes de ver los contenidos del curso y luego de su ejecución, se realizó la misma evaluación. A continuación el resultado cuantitativo de la emisión del curso.

Tabla 3. Resultados cuantitativos del curso virtual masivo

\begin{tabular}{|l|c|}
\hline Alumnos Matriculados & 542 \\
\hline Alumnos Que Ingresaron Al Curso & 321 \\
\hline Promedio Antes Del Curso & $62,89 / 100$ \\
\hline Promedio Después Del Curso & $76,32 / 100$ \\
\hline Alumnos Que Mejoraron El Conocimiento & 246 \\
\hline
\end{tabular}

Fuente: Elaboración propia.

\section{CONCLUSiOnes}

- La capacitación virtual permite acortar distancias, esto quiere decir que la MOOC puede ser ejecutada en cualquier parte del país y del mundo.

- Las Mooc solventan problemas de horario y de grandes laboratorios para recibir capacitación presencial

- La adaptabilidad para móviles es muy importante porque la nueva tendencia tecnológica está orientada a estos dispositivos.

- La capacitación en general es parte de una cultura orientada la prevención de accidentes de tránsito.

- Los efectos de la capacitación sobre el índice de accidentes de tránsito será apreciable de un periodo de ejecución no menor a 5 años de manera permanente.

\section{RECOMENDACIONES}

- Difundir la Mooc a través de los diferentes canales de CEDIA para que esta llegue a todo el mundo.

- Fomentar el uso de la MOOC en entidades educativas a todo nivel

Socializar el uso de la MOOC en entidades relacionadas con el manejo de actividades de tránsito.

\section{REFERENCIAS BIBLIOGRÁFICAS}

ANT (2014). Agencia Nacional de Tránsito.

Bartolomé, A. (2004). Blended learning. conceptos básicos. universidad de barcelona.

del Rosario Sánchez, M., Miguel, V., Díaz, K., Vílchez, G., Villasmil, S., and López, M. G. (2009). Entorno virtual de enseñanza-aprendizaje para la construcción del conocimiento en bioquímica médica. Revista de la Facultad de Medicina, 32(1):31-37.

La Hora (2013). Diario La Hora.

OMS, O. M. d. 1. S. (2009). Informe sobre el estado de la seguridad vial en la región de las américas.

OPS, O. P. d. 1. S. (2011). Traumatismos causados por el tránsito y discapacidad.

Rosenberg, M. J. (2001). E-learning: Strategies for delivering knowledge in the digital age, volume 3. McGraw-Hill New York.

Universidad Carlos III (2009). El origen del MOOC.

William, R. and Rice, N. (2009). Moodle, desarrollo de cursos e-learning. Editorial Agapea, Madrid-España. 